\title{
Value of cerebrospinal fluid examination in the diagnosis of meningitis in the newborn
}

\author{
Lidia Hristeva, Ian Bowler, Robert Booy, Andrea King, Andrew R Wilkinson
}

\begin{abstract}
Between 1 October 1988 and 30 September 1991 the results of all 896 cerebrospinal fluid examinations from 736 neonates were correlated with clinical diagnosis, treatment, and outcome. The prevalence of fungal or bacterial meningitis in babies requiring lumbar puncture was only $0.95 \%$. Gram staining had a sensitivity of $68 \%$ and a positive predictive value of only $46 \%$ for the diagnosis of meningitis. Primary cultures directly onto agar plates had a sensitivity of $81 \%$ and positive predictive value of $46 \%$. Broth enrichment cultures did not improve sensitivity and were frequently found to be false positive. Empirical treatment should not be altered unless more than a few organisms are seen on Gram staining. Primary cultures are adequate for the diagnosis of fungal and bacterial meningitis. Enrichment cultures should be performed only when the Gram stain and/or cell count suggests meningitis is likely. Clinicians should be aware that diagnostic tests performed in populations with a low prevalence of disease are likely to generate many false positive results and have a low positive predictive value.

(Arch Dis Child 1993; 69: 514-517)
\end{abstract}

When systemic infection is suspected in the newborn it is usual to carry out a lumbar puncture to exclude meningitis. The procedure is not without risk and possible complications include acute respiratory compromise, trauma, introduction of infection, spinal epidermoid implantation tumours, and contamination of cerebrospinal fluid (CSF) with bone marrow cells. $^{1-6}$ We wished, therefore, to assess the utility of Gram stain, primary culture, and enrichment culture in establishing the diagnosis of bacterial and fungal meningitis in babies admitted to the special care baby unit at the John Radcliffe Maternity Hospital, Oxford.

John Radcliffe Hospital, Headington, Oxford OX3 9DU, Infectious Disease and Neonatal Units, University Department of Paediatrics Lidia Hristeva Robert Booy Andrew R Wilkinson

Public Health Laboratory Ian Bowler Andrea King

Correspondence to: Dr Bowler.

Accepted 23 July 1993 lumbar puncture after the neonatal period $(>28$ days old) were classified separately. The unit has a policy of delaying lumbar puncture in small preterm infants with respiratory distress because of the risk of intraventricular haemorrhage in the first two days after birth. ${ }^{7}$ During the review period the clinical course, treatment, and outcome of all neonates with suspected infection had been prospectively recorded and cases of meningitis noted.

Using data recorded concurrently in the bacteriology department the results of all CSF examinations performed on neonates during this review period were analysed. Details recorded were adequacy of the sample, white cell count, red cell count, Gram stain, and culture result. Primary culture was performed by inoculating one drop of the centrifuged deposit of CSF onto each of three agar plates: chocolate agar and 5\% horse blood agar plates (incubated in air $+5 \%$ carbon dioxide) and a second 5\% horse blood agar plate (incubated anaerobically). All plates were incubated for 48 hours at $37^{\circ} \mathrm{C}$. Enrichment culture was performed by adding a drop of the deposit to a tryptose soy broth which was routinely subcultured onto agar plates after three days incubation or before this if growth was visible.

In addition, all cases of neonatal meningitis and their corresponding lumbar puncture results from 1 May 1984 to 30 September 1988 were available for analysis from a prospectively recorded database. It was possible, therefore, to review the medical notes of all infants diagnosed with meningitis during that period. Cases of definite bacterial or fungal meningitis were defined by a suggestive clinical presentation, for example thermal and respiratory instability, feed intolerance and/or seizures, supported by positive primary culture or postmortem evidence if a lumbar puncture had not been carried out. Cases of probable meningitis were defined by suggestive clinical features together with either a positive enrichment culture or, if the culture was negative, a positive Gram stain or high white cell count $\left(>100 \times 10^{9} / 1\right)$. All such cases received appropriate antibiotic treatment for meningitis $(>10$ days intravenous treatment). A false positive (contaminated) culture was defined by the growth of an organism without CSF pleocytosis or positive Gram stain together with a clinical picture which did not suggest meningitis. Such cases all received less than five days intravenous antibiotics. A traumatic blood stained CSF sample was defined using the criteria of Visser and Hall, namely a red cell count greater than $10 \times 10^{9} / .^{8}$

The Epi-Info version 5 was used for statistical analysis (Centers for Disease Control, Atlanta). The $\chi^{2}$ test with Yates's correction

dod


Table 1 Distribution of lumbar punctures by age and indication

\begin{tabular}{|c|c|c|c|}
\hline $\begin{array}{l}\text { Age } \\
\text { (days) }\end{array}$ & $\begin{array}{l}\text { No of } \\
\text { babies }\end{array}$ & $\begin{array}{l}\text { No of lumbar } \\
\text { punctures }\end{array}$ & $\begin{array}{l}\text { Lumbar } \\
\text { punctures/ } \\
\text { patients }\end{array}$ \\
\hline$\leqslant 28$ & 736 & 896 & $1 \cdot 2$ \\
\hline $\begin{array}{l}\text { Early infection } \\
(\leqslant 48 \text { hours })\end{array}$ & $564^{\star}$ & 586 & $1 \cdot 0$ \\
\hline $\begin{array}{c}\text { Late infection } \\
(>48 \text { hours })\end{array}$ & $225^{\star}$ & 310 & $1 \cdot 3$ \\
\hline$>28$ & $63 t$ & 166 & $2 \cdot 6$ \\
\hline Total & 760 & 1062 & $1 \cdot 4$ \\
\hline
\end{tabular}

^Fifty three infants had a lumbar puncture for both suspected early and late onset infection.

†Twenty four infants did not also have a lumbar puncture during neonatal period.

was employed in comparisons, unless an expected cell value was $<5$ when Fisher's exact test (two tailed) was employed.

\section{Results}

(A) FREQUENCY OF CSF EXAMINATION

Between 1 October 1988 and 30 September 1991, 19491 babies were born at the John Radcliffe Maternity Hospital, and of these $1625(8 \cdot 3 \%)$ were admitted to the unit. A further 178 babies were referred from other centres. Of the 1803 babies admitted to the unit, $760(42 \%)$ underwent lumbar puncture. Table 1 summarises the distribution of lumbar punctures by age and indication. Table 2 summarises the birth weight and gestational age of the 896 neonates who required the procedure.

(B) ADEQUACY OF SAMPLE

Data recorded on adequacy of sample is presented in table 3. One third of all samples were unsuitable for full cytological analysis because the sample was grossly bloodstained, clotted, or too small.

(C) VALUE OF GRAM STAINING

Between 1 October 1988 and 30 September 1991 five cases of definite bacterial meningitis (three early, two late), one case of probable bacterial meningitis (late), and one case of definite candida meningitis (late) were diagnosed. The prevalence of bacterial and fungal meningitis in neonates undergoing lumbar puncture in our unit was $0.95 \%(7 / 736)$. There was a higher yield of true positives from late lumbar puncture $(4 / 310,1 \cdot 3 \%)$ compared with early $(3 / 586,0.5 \%)$ but the difference was not statistically significant.

Table 2 Gestational age and birth weight of neonates undergoing lumbar puncture

\begin{tabular}{|c|c|c|c|c|c|c|}
\hline & \multicolumn{3}{|c|}{ Early ( $\leqslant 48$ hours) } & \multicolumn{3}{|c|}{ Late ( $>48$ hours) } \\
\hline & $\begin{array}{l}\text { No (\%) } \\
\text { babies }\end{array}$ & $\begin{array}{l}\text { No of } \\
\text { lumbar } \\
\text { punctures }\end{array}$ & $\begin{array}{l}\text { Lumbar } \\
\text { punctures/ } \\
\text { patient }\end{array}$ & $\begin{array}{l}\text { No (\%) } \\
\text { babies }\end{array}$ & $\begin{array}{l}\text { No of } \\
\text { lumbar } \\
\text { punctures }\end{array}$ & $\begin{array}{l}\text { Lumbar } \\
\text { punctures/ } \\
\text { patients }\end{array}$ \\
\hline $\begin{array}{l}\text { Birth weight }(\mathrm{g}) \\
<1500 \\
1500-2500\end{array}$ & $\begin{array}{c}45(8) \\
188(33)\end{array}$ & 46 & $1 \cdot 0$ & $\begin{array}{r}105(47) \\
48(21)\end{array}$ & 176 & $1 \cdot 7$ \\
\hline $\begin{array}{l}>2500 \\
\text { Gestational age } \\
\text { (weeks) }\end{array}$ & $331(59)$ & 540 & $1 \cdot 0$ & $72(32)$ & 134 & 1.1 \\
\hline $\begin{array}{l}<31 \\
\geqslant 31\end{array}$ & $\begin{array}{c}32(6) \\
532(94)\end{array}$ & $\begin{array}{r}33 \\
553\end{array}$ & $\begin{array}{l}1 \cdot 0 \\
1 \cdot 0\end{array}$ & $\begin{array}{r}88(39) \\
137(61)\end{array}$ & $\begin{array}{l}154 \\
156\end{array}$ & $\begin{array}{l}1 \cdot 8 \\
1 \cdot 1\end{array}$ \\
\hline Total & 564 & 586 & $1 \cdot 0$ & 225 & 310 & $1 \cdot 4$ \\
\hline
\end{tabular}

Table 3 Adequacy of lumbar puncture from neonates for cytological analysis

$\left.\begin{array}{lccc}\hline & \text { Totals } & \multicolumn{2}{c}{\text { No (\%) }} \\ \hline & & \multicolumn{2}{c}{\text { Inadequate }} \\ \text { All } & 896 & 299 & (33) \\ \text { Early } & 586 & 189 & (32) \\ \text { Late } & 310 & 110 & (35) \\ & & \multicolumn{2}{c}{\text { Bloodstained }} \\ \text { All } & 896 & 243 & (27) \\ <1500 \mathrm{~g} & 222 & 72 \\ \geqslant 1500 \mathrm{~g} & 674 & 171\end{array}\right\} \star(25)$

Gram staining was carried out on all 896 CSF specimens sent from neonates and was positive in 13, six of which were considered true positives and seven false positives. In all the false positive Gram stains, only scanty organisms were seen. There was only one false negative Gram stain result which was from the child with candida meningitis. Thus, the sensitivity of Gram staining was $86 \%$, specificity $99 \%$, positive predictive value $46 \%$, and the negative predictive value $99 \cdot 9 \%$.

Between 1 May 1984 and 30 September 198810 other cases of bacterial or fungal meningitis were recorded. One case was diagnosed only at postmortem examination. In five of the nine cases diagnosed by lumbar puncture organisms were seen on Gram staining giving a sensitivity for the test for the combined review periods of $68 \%(5+6 / 16)$.

\section{(D) VALUE OF PRIMARY CULTURE}

Primary culture was performed on all 896 specimens. A total of 13 positive results were obtained. Of these 13 results, seven were considered false positive and the organisms isolated are listed in table 4 . There was no overlap between the false positive cultures and the false positive Gram stain results. There was only one false negative primary culture result, in a baby with a probable late onset meningitis. Gram negative rods were seen in the stained deposit but the organisms failed to grow on both primary and enrichment culture. The baby had received antibiotics before lumbar puncture. Details of the six true positive

Table 4 Contaminants isolated on primary or enrichment culture (false positive) from 108 lumbar punctures

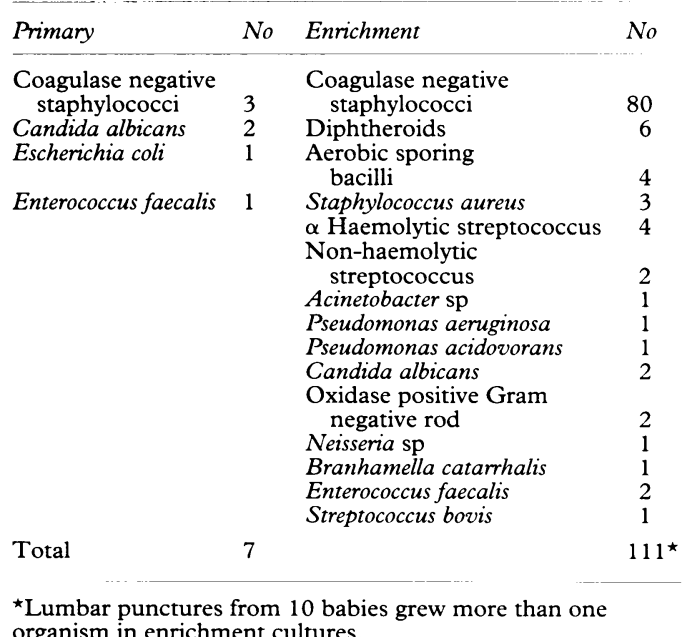


Table 5 Organisms isolated from cases of meningitis

\begin{tabular}{|c|c|c|}
\hline & Definite & Probable \\
\hline $\begin{array}{l}1 \text { October } 1988 \text { - } 30 \text { September } 1991 \\
\text { Group B streptococcus } \\
\text { Serratia liquifaciens } \\
\text { Candida albicans }\end{array}$ & $\begin{array}{l}4 \\
1 \\
1\end{array}$ & \\
\hline $\begin{array}{l}1 \text { May } 1984 \text { - } 30 \text { September } 1988 \\
\text { Group B streptococcus } \\
\text { Klebsiella pneumoniae } \\
\text { Klebsiella oxytoca } \\
\text { Pseudomonas aeruginosa } \\
\text { Listeria monocytogenes } \\
\text { Streptococcus mitis } \\
\text { Achromobacter xylosoxidans } \\
\text { Candida albicans }\end{array}$ & $\begin{array}{l}1 \\
1 \\
1\end{array}$ & 1 \\
\hline
\end{tabular}

culture results are given in table 5 . The sensitivity of primary culture was $86 \%$ with a specificity of $99 \%$, the positive predictive value was $46 \%$, and the negative predictive value $99.9 \%$.

Of the nine cases of meningitis diagnosed by lumbar puncture recorded between 1 May 1984 and 30 September 1991, seven were positive on primary culture. Thus for the combined review periods the sensitivity of primary culture was $81 \%(6+7 / 16)$.

\section{(E) VALUE OF ENRICHMENT CULTURE}

The value of enrichment culture was also assessed. Enrichment was performed on all 896 lumbar puncture specimens and these were positive in 101. All were false positive. Combining false positive primary and enrichment cultures there were therefore 108 contaminated cultures, and these were more often seen when the lumbar puncture was done for possible late sepsis $(53 / 310,17 \%)$ than in those done early $(55 / 586,9 \cdot 4 \%, \mathrm{p}=0.001)$. There was no evidence that lumbar punctures done in babies with a low birth weight $(<1500 \mathrm{~g})$ were more likely to be contaminated (early 4/46, 9\% and late 28/176, 16\%) than those done in larger babies (early $51 / 540$, $9 \%$ and late $25 / 134,18 \cdot 7 \%$ ). Table 4 summarises the identity of organisms grown from contaminated cultures. Coagulase negative staphylococci (CoNS) were by far the most common isolates. There were 12 neonates from whom CoNS were isolated from blood and CSF simultaneously. In each case the isolate from the CSF was distinguishable from that in the blood by antibiogram (differing by three or more sensitivity tests from a panel of 12). Three of these infants were treated for CoNS septicaemia for between nine and 13 days; the CSF isolates were regarded as contaminants. In the other nine cases the CoNS in both the blood and the CSF were regarded as contaminants. CoNS were not isolated from subsequent CSF samples obtained from these babies and none developed a clinical picture suggestive of meningitis.

In the light of the apparent lack of value of enrichment culture, we reviewed the contribution of this procedure in the diagnosis of cases of meningitis recorded in the period 1 May 1984 to 30 September 1988 . Of the nine cases diagnosed antemortem by lumbar puncture two grew organisms only on enrichment. Both had been classified as probable cases.

\section{Discussion}

Neonatal meningitis is uncommon. The incidence of $0 \cdot 36 / 1000$ live births/year which we observed in Oxford is comparable with a national figure of $0 \cdot 32 / 1000 /$ year reported for England and Wales. ${ }^{9}$ The proportion of babies admitted to the unit who underwent lumbar puncture is high $(42 \%)$. This is a reflection of the difficulty of making the diagnosis of meningeal infection on clinical grounds in sick neonates. Thus lumbar puncture is performed as a screening procedure in a population with known risk factors for meningitis but where the prevalence of the disease is low $(0.95 \%)$. Under these conditions there is a need for a test with high sensitivity. However, attempts to increase the sensitivity of a test may lead to a fall in specificity. One third of samples in our study were inadequate for cytological assessment either because they were too small or grossly bloodstained. Others have found that between 15 and $50 \%$ of samples are unsuitable in this way. ${ }^{810}$ Furthermore up to $15 \%$ of neonates may have bacterial meningitis in the absence of a CSF pleocytosis. ${ }^{8}$ Thus greater emphasis must be placed on the results of Gram stain and culture.

Gram staining is a less sensitive method than culture for detecting micro-organisms. The sensitivity of $68 \%$ for the two review periods is acceptable, given the low prevalence of meningitis in the study population. This compares well with the $80 \%$ sensitivity observed in studies of childhood meningitis where the prevalence of the disease is much higher. ${ }^{11} 12$ The two cases of candida meningitis in the combined review periods both generated false negative Gram stain results. This emphasises the difficulty of diagnosing this condition rapidly. ${ }^{13}$ However, a negative Gram stain in a neonatal lumbar puncture should reassure the clinician as this has a high negative predictive value $(99 \cdot 9 \%)$. The low positive predictive value of Gram staining ( $46 \%$ ) is a consequence of the low prevalence of meningitis in this population. In all false positive Gram stains very few organisms were seen on the film. Specificity could be improved if these were not reported. However, considering the 16 cases of meningitis diagnosed by lumbar puncture in the combined review periods, very few organisms were seen on films in three cases of true meningitis and therefore sensitivity would suffer. No particular morphological type was associated with false positivity or true positivity. The causes of false positive Gram stains include contamination of specimen tubes, glass slides and Gram reagents, ${ }^{14}$ and the epithelial cells from the child's skin. ${ }^{15}$ However, this is the first study to quantify the size of the problem in a large series of tests.

The interpretative value of primary culture is similar to Gram staining, though culture is more sensitive $(81 \%)$. Though the positive predictive value is low $(46 \%)$, interpretation is made easier by the amount of growth and the identity of the organism. Cultures growing group B streptococci were always significant. Cultures yielding CoNS were, in our series, always contaminants. The significance of 
aerobic Gram negative rods and candida was difficult to assess as these were found as both contaminants and significant cultures. We observed a non-significant trend towards an increased yield of true positives from late lumbar punctures $(4 / 310,1.3 \%)$ compared with early $(3 / 586,0.5 \%)$. This has also been observed by others and may reflect different indications for lumbar puncture in the two groups, maternal reasons being more important for suspected early onset infection. ${ }^{10}$

We have not evaluated the effect of antibiotic treatment given during labour. A recent review of practice in the USA showed that $8.8 \%$ of babies had been exposed to antibiotics in this way. ${ }^{10}$ This may influence the yield of cultures from early lumbar punctures. However, we found only one baby with partially treated meningitis, and this child's cultures were negative because he had himself received antibiotics. Partially treated meningitis appears to be uncommon in neonatal units. $^{810}$ This may be because lumbar puncture is usually done before antibiotics are started and/or because the high proportion of inadequate specimens means that if cultures are negative, no further interpretation can be made.

Enrichment culture is designed as an exquisitely sensitive technique to resuscitate organisms damaged by the host response and antibiotic treatment. In the second review period (1988-91) 896 enrichments were performed generating 101 false positives without improving on the sensitivity of primary culture. Combining the two review periods and given an annual lumbar puncture rate of about 300 a year, two cases of probable meningitis were detected by an estimated 2225 enrichments which would have generated an estimated 240 false positives. There are few data available on specificity of CSF culture as most series exclude false positive results, ${ }^{11} 12$ yet such data are vital to the doctor at the bedside who is attempting to interpret the clinical significance of the result. A recent review of practice in a neonatal unit in the USA where the laboratory used equivalent techniques to our own, showed that of 1104 lumbar punctures performed in 826 babies only 17 cultures were positive and of these 12 were considered false positives. ${ }^{10}$ The identity of organisms grown on enrichment may help with interpretation as most are derived either from skin (CoNS and diphtheroids) or the environment (aerobic sporing bacilli, Pseudomonas sp). However, most of these organisms have also been described as rare causes of meningitis in neonates and the generation of so many false positives may lead to diagnostic uncertainty, repeat lumbar punctures, and unnecessary antibiotic treatment. The origin of these contaminants is uncertain.
They could arise at both the bedside and/or during handling in the laboratory.

In conclusion, because lumbar puncture is performed in neonates as a screening procedure, the bacteriological tests carried out behave quite differently when compared with the same tests performed on CSF from adults or children suspected to have meningitis where the prevalence of the disease being sought is greater. The positive predictive value of Gram staining in the neonatal lumbar puncture is so low that unless more than a few organisms per several high power fields are visualised empirical antibiotic treatment should not be altered on the basis of this test alone. Primary cultures have adequate sensitivity, and though positive predictive value is low, the identity of organisms aids interpretation. To perform enrichment culture on all lumbar punctures from neonates does not seem cost effective. It should be confined to samples from babies who have already received antibiotics, or where organisms are seen on the Gram stain, or where there is a raised white blood cell count in the CSF. Clinicians should be aware that diagnostic tests performed in populations with a low prevalence of disease are likely to generate many false positive results and have a low positive predictive value. Systematic review of the performance of laboratory tests against clinical gold standards leads to more rational use of laboratory resources and improves interpretation at the bedside.

1 Batnitzny S, Kencher TR, Mealey J Jr, Campbell RL. Iatrogenic intraspinal epidermoid tumours. $\mathfrak{F} A M A 1977$; 237: $148-50$.

2 Teele DW, Dashefsky B, Rakustom T, Klein JO. Meningitis after lumbar puncture in children with bacteraemia. $N$ Engl f Med 1981; 305: 1079-81.

3 Krusnall MS, Carter SR, Ritz LP. Contamination of cerebrospinal fluid by vertebral bone marrow cells during lumbar puncture. N Engl f Med 1983; 308: 697-700.

4 Long JG, Phillip AGS, Lucey JF. Excessive handling as a cause of hypoxemia. Pediatrics 1980; 65: 203-7.

5 Speidel BD. Adverse effects of routine procedures on Speidel BD. Adverse effects of routin
preterm infants. Lancet 1978; i: 864-5.

preterm infants. Lancet 1978; i: 864-5.
6 Weisman LE, Merenstein GB, Steenberger JR. The effect of Weisman LE, Merenstein GB, Steenberger JR. The effect of
lumbar puncture position in sick neonates. Am $\mathcal{J}$ Dis Child 1983; 137: 1077-9.

7 Szymonowicz W, Yu VYH. Timing and evolution of periventricular haemorrhage in infants weighing $1250 \mathrm{~g}$ or less at birth. Arch Dis Child 1984; 59: 7-12.

8 Visser VE, Hall RT. Lumbar puncture in the evolution of suspected neonatal sepsis. $\mathcal{F}$ Pediatr 1980; 96: 1063-7.

9 de Louvois J, Blackburn J, Hurley R, Harvey D. Infantile meningitis in England and Wales: a two year study. Arch Dis Child 1991; 66: 603-7.

10 Schwersenski J, McIntyre L, Bauer CR. Lumbar puncture frequency and cerebrospinal fluid analysis in the neonate. frequency and cerebrospinal fluid

11 Marks WA, Stutman HR, Marks MI, et al. Cefuroxine versus ampicillin plus chloramphenicol in childhood bacterial meningitis: a multicenter randomised trial. $f$ Pediatr 1986; 109: 123-30.

12 Schaad U, Suter S, Gianella A, et al. A comparison of ceftriaxone and cefuroxime for the treatment of bacterial meningitis in children. N Engl f Med 1990; 322: $141-7$

13 Phillips G, Colledge C. Fungal infections in neonates. $f$ Antimicrob Chemother 1991; 28: 159-61.

14 Musher MD, Schell RF. False positive Gram stains of cerebrospinal fluid. Ann Intern Med 1973; 79: 603-4.

15 Joyner RW, Idriss ZH, Wilfert CM. Misinterpretation of cerebrospinal fluid Gram stain. Pediatrics 1974; 4: 360-2. 